\title{
Erratum to: Fisetin yeast-based bio-capsules via osmoporation: effects of process variables on the encapsulation efficiency and internalized fisetin content
}

\author{
Antonio de Anchieta Câmara Jr. ${ }^{1,4}$ - Sébastien Dupont ${ }^{4} \cdot$ Laurent Beney ${ }^{4}$. \\ Patrick Gervais ${ }^{4}$ - Amauri Rosenthal ${ }^{3}$ - Roberta Targino Pinto Correia ${ }^{2}$. \\ Márcia Regina da Silva Pedrini ${ }^{1}$
}

Published online: 29 March 2016

(C) Springer-Verlag Berlin Heidelberg 2016

Erratum to: Appl Microbiol Biotechnol

DOI 10.1007/s00253-016-7425-8

The original version of this article inadvertently contained a mistake for the author named "Antonio Anchieta de Câmara Jr.”.

Full name: Antonio de Anchieta Câmara Jr.

Citation: Câmara Jr., A. A.

The online version of the original article can be found at http://dx.doi.org/ $10.1007 / \mathrm{s} 00253-016-7425-8$

Antonio de Anchieta Câmara, Jr.

antonio_camara-junior@etu.u-bourgogne.fr

$\triangle$ Márcia Regina da Silva Pedrini

marcia.pedrini@eq.ufrn.br

1 Bioprocess Laboratory, Chemical Engineering Department, Federal University of Rio Grande do Norte, Lagoa Nova,

Natal, RN 59078-900, Brazil

2 Laboratory of Bioactive Compounds, Chemical Engineering Department, Federal University of Rio Grande do Norte, Lagoa Nova, Natal, RN 59078-900, Brazil

3 Embrapa Food Technology, Av. das Américas, 29501, Rio de Janeiro, RJ 23020-470, Brazil

4 UMR Procédés Alimentaires et Microbiologiques (PAM), Univ. de Bourgogne Franche-Comté/AgroSup Dijon, 1 esplanade Erasme, 21000 Dijon, France 